\title{
Rapid switching in a dual-frequency hybrid aligned nematic liquid crystal cell
}

\author{
S. A. Jewell, ${ }^{\text {a) }}$ T. S. Taphouse, and J. R. Sambles \\ Thin Film Photonics Group, School of Physics, University of Exeter, Stocker Road, Exeter, EX4 4QL, \\ United Kingdom
}

(Received 14 March 2005; accepted 27 May 2005; published online 8 July 2005)

\begin{abstract}
We report the optical characterization of a dual-frequency hybrid aligned nematic (HAN) liquid crystal cell driven between two states using a multiple-frequency sinusoidal pulse. The complex dynamic director structure formed during the fast switching process is resolved in unprecedented detail on a submillisecond time scale. The results reveal backflow effects and a total switching time that is substantially faster than that achievable with conventional HAN cells. (C) 2005 American Institute of Physics. [DOI: 10.1063/1.1992672]
\end{abstract}

To allow liquid crystal devices to maintain their position as the primary flat-panel display, the quality of video rate images, dominated by the switching speed of the pixels, must be improved. This fundamental limitation is dictated by the viscoelastic properties of the liquid crystal, and to overcome this problem both the structure of the liquid crystal cell and the material itself must be considered.

One potential fast-switching structure is the hybrid aligned nematic (HAN) cell, which has already been demonstrated as a commercially significant geometry in the new generation of zenithal bistable devices. ${ }^{1}$ A HAN cell has homogeneous alignment on one surface and homeotropic alignment on the other. To a first approximation, this geometry offers equal bias to both homogeneous and homeotropic alignment, resulting in a near-linear variation in tilt with distance through the cell. On application of a voltage across a HAN cell, a thresholdless distortion can readily be produced, aligning the director either homeotropically or homogeneously depending on the sign of the dielectric anisotropy of the nematic liquid crystal used. ${ }^{2,3}$ An interesting possibility arises when a HAN structure is combined with a dualfrequency liquid crystal; ${ }^{4,5}$ these materials have a high dielectric dispersion where the dielectric anisotropy, $\Delta \varepsilon(f)$ $=\varepsilon_{\|}(f)-\varepsilon_{\perp}(f)$, is frequency dependent, resulting in a change in sign at the crossover frequency $f_{\text {co }}$, where $\Delta \varepsilon\left(f_{\text {co }}\right)=0$. Certain dual-frequency materials are commercially available in which $f_{\text {co }}$ occurs at a few $\mathrm{kHz}$ and $\Delta \varepsilon(f)$ changes significantly over the range $1-100 \mathrm{kHz}$. In a dual-frequency HAN cell, the director can be driven between either homogeneous or homeotropic alignment with equal ease by applying an electric field across the sample at a frequency either above or below $f_{\text {co }}{ }^{6}$ This provides a relatively straightforward means of switching the cell between the birefringent homogeneous alignment and the uniaxial homeotropic alignment, which are both optically very different from the relaxed $0 \mathrm{~V}$ state.

Optical waveguide techniques have become one of the leading tools for the detailed measurement of the director profiles in numerous liquid crystal structures. ${ }^{7-9}$ A modification to the synchronization of voltage application and data collection in the standard fully leaky guided mode (FLGM) technique ${ }^{10}$ has now been implemented, allowing the director orientation during dynamic reorientation to be recorded on

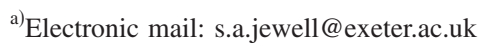

an unprecedented $2 \mu$ s time scale. This time-resolved FLGM technique maintains extreme sensitivity to subtle director changes associated with the original FLGM technique used for static measurements. Here it has been used to measure the dynamic response of such a dual-frequency HAN cell when a multiple pulse is used to switch the cell from $0 \mathrm{~V}$ to homeotropic alignment and then driven back to the $0 \mathrm{~V}$ state.

A HAN cell of thickness $4.85 \mu \mathrm{m}$ was constructed using indium tin oxide (ITO)-coated glass substrates. To produce the required alignment, the upper and lower substrates were separately treated with homogeneous and homeotropic polyimides, respectively, and were spaced around the edges using glass beads dispersed in UV-setting glue. The cell was then filled in the isotropic phase with the dual-frequency nematic liquid crystal MDA-00-3969 (Merck Kga), cooled to room temperature $\left(19.5^{\circ} \mathrm{C}\right)$, and sealed. The sample was then sandwiched between two $60^{\circ}$ low index glass prisms ( $n$ $=1.52$ ) index matched to the cell substrates and the whole assembly was mounted at the center of a rotating table (Fig. 1) to allow optical intensity versus angle-of-incidence data to be collected in reflection and transmission for $p$ - (transverse magnetic) and $s$ - (transverse electric) polarized light. At each angle-step, a pulsed voltage was applied to the cell and the optical intensity data corresponding to the reorientation of the director during the pulse was captured on a $2 \mu$ s time scale. The data-capture process was synchronized with the application of the voltage, allowing precise information about the optical and hence physical response of the cell during the entire switching process to be obtained. At each angle-step, the pulse used consisted of an initial burst of a

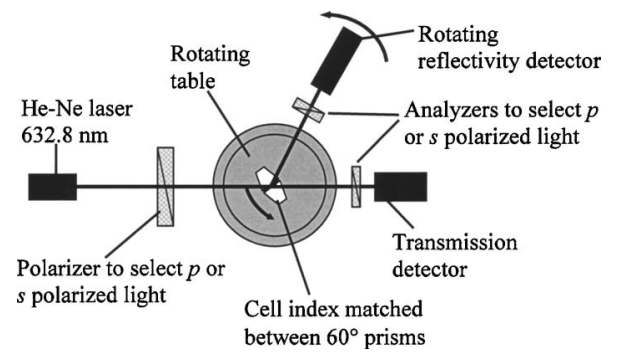

FIG. 1. Experimental arrangement of the time-resolved fully leaky guidedmode technique. The application of the voltage pulse from a function generator and the data collection at the transmission and reflectivity detectors are synchronized and controlled through a LABVIEW interface. 


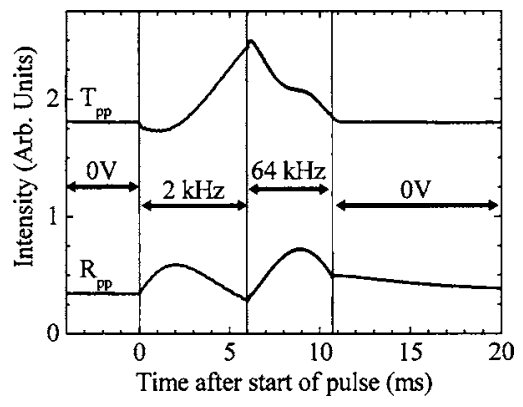

FIG. 2. Variation of optical intensity with time for transmitted and reflected $p$-polarized conserving data at a $74^{\circ}$ angle of incidence. The data were collected on a $2 \mu$ s time scale during the application of a $20 \mathrm{~V}_{\mathrm{pp}}$ multiplefrequency sinusoidal pulse as indicated.

$2 \mathrm{kHz} 20 \mathrm{~V}_{\mathrm{pp}}$ sinusoidal voltage for $6.0 \mathrm{~ms}$, immediately followed by a sinusoidal voltage at $64 \mathrm{kHz} 20 \mathrm{~V}_{\mathrm{pp}}$ for $4.8 \mathrm{~ms}$, and then the voltage was returned to $0 \mathrm{~V}$ (short circuit) for the remainder of the data collection at that angle of incidence. A typical optical response of the cell at a single angle of incidence is shown in Fig. 2. The duration and frequencies of the pulses used here were selected to give the shortest combined time to switch from the initial $0 \mathrm{~V}$ state to a static almost purely homeotropic state and back to the $0 \mathrm{~V}$ state. This total switching time was determined by measuring the amount of time between the start of the pulse being applied and the point at which the transient optical response of the cell after the removal of the voltage reaches a point that is not discernable from the $0 \mathrm{~V}$ state. It was found that this apparent end-point varied with the angle of incidence since the optical response at different angles is sensitive to different regions through the cell. ${ }^{11}$ The optical data was thus collected over a wide range of angles, with the higher angles of incidence (associated with the higher-momentum modes) showing the greatest sensitivity to changes in the cell refractive index and hence director orientation. Due to the sensitivity of the dielectric anisotropy of the cell to changes in temperature, the temperature was monitored throughout the experiment and the duration over which voltages were applied to the cell were kept to a minimum to prevent heating of the material.

To obtain the director profiles during the reorientation process, the optical data for each polarization combination were separated into individual time steps. All eight datasets for each time were then fitted to simultaneously using a multilayer optics model based on a $4 \times 4$ Berreman matrix ${ }^{12}$ with the best-fit determined from a least-squares fitting routine. As the optical parameters for the ITO, polyimides, and liquid crystal had been previously determined, these were fixed, and only the tilt profile (described mathematically by a Bezier function to allow good control over the start and end tilt points, as well as the form of the profile in the centre of the cell) was allowed to vary. Fitting to multiple polarization datasets simultaneously removed any degeneracy from the fitting procedure. The reflected intensity data was particularly sensitive to the director tilt in the first half of the cell, producing an uncertainty in the tilt in this region of less than $1^{\circ}$.

The resulting tilt profiles are shown in Fig. 3. The initial $6 \mathrm{~ms}$, where the cell is responding to the application of the $2 \mathrm{kHz}$ voltage [Fig. 3(a)] is similar in form to the switch-on profile associated with a HAN cell filled with a standard positive dielectric anisotropy liquid crystal. ${ }^{13}$ The total re-
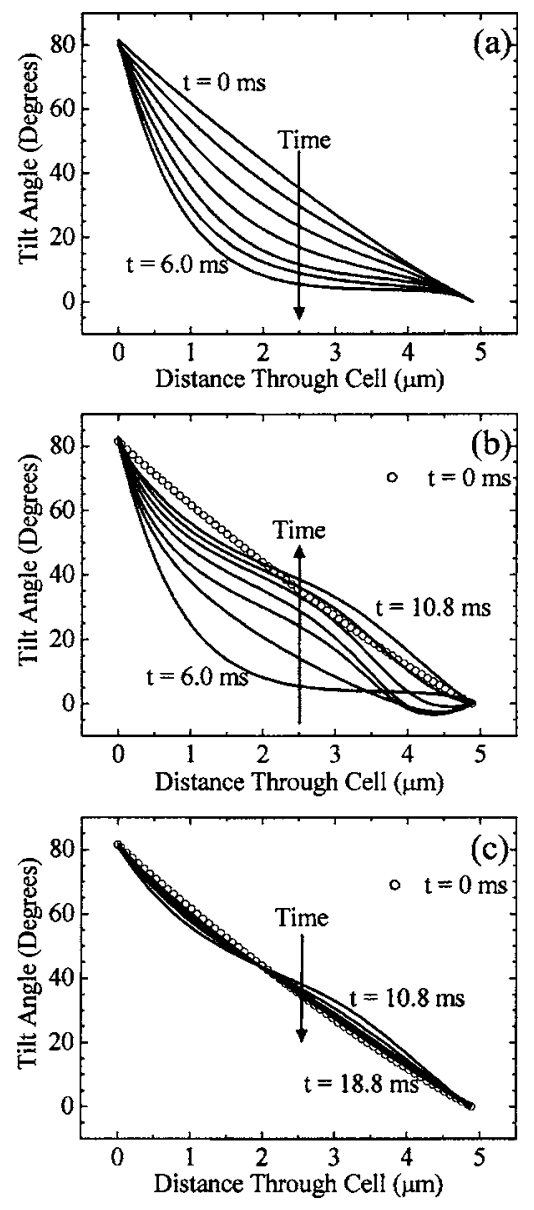

FIG. 3. The tilt profiles (with the tilt angle measured from the normal to the cell substrate) recorded during the application of the multiple-frequency pulse shown in Fig. 2: (a) during the application of the $2 \mathrm{kHz}$ in $1 \mathrm{~ms}$ steps, (b) during the application of the $64 \mathrm{kHz}$ pulse in $0.8 \mathrm{~ms}$ steps, and (c) with the voltage removed in $2 \mathrm{~ms}$ steps. In (b) and (c) the initial director profile at $t=0 \mathrm{~ms}$ is shown for reference.

sponse time for the cell to reach equilibrium is adversely affected by the relatively small value of the dielectric permittivity of the material at that frequency $[\Delta \varepsilon(2 \mathrm{kHz}) \approx 2.0] \mathrm{re}-$ sulting in a small torque on the director. This also reduces any influence of a coupling between rotation and flow of the molecules, so that backflow at the homeotropic surface, which is prevalent in the switch-on of a ZLI-2293 filled HAN cell, ${ }^{13}$ is not seen. On application of the highfrequency section of the pulse $[\Delta \varepsilon(64 \mathrm{kHz}) \approx-1.0]$ the cell shows an immediate response to the change in frequency corresponding to the change in the sign of the dielectric permittivity of the liquid crystal. The immediate response of the cell to the change in frequency within one cycle of the highfrequency sine wave highlights how the dual-frequency material responds to the rate of change of the applied field. In particular this extreme sensitivity to the shape of the leading edge of the pulse rather than a response to the net field indicates that when analyzing the dynamic response of the material the Fourier components of the applied field should be included in the frequency-dependent dynamic model.

Fitting to the high-frequency optical data shows that initially the main bulk of the cell responds by becoming increasingly homogeneously aligned [Fig. 3(b)]. However, at the homeotropic surface backflow can clearly be seen occurring during the initial $2 \mathrm{~ms}$ of the $4.8 \mathrm{~ms}$ high-frequency pulse. In this region, a coupling between the rotation and
AIP license or copyright; see http://apl.aip.org/apl/copyright.jsp 
flow in the liquid crystal causes the director to overrotate in the opposite direction to the main bulk of the cell before reversing direction and being driven towards homogeneous alignment. After this time the whole cell is uniformly driven towards homogeneous alignment for the remainder of the pulse duration. At the point at which the voltage is removed completely, the director is close to the near-linear profile of the $0 \mathrm{~V}$ state, although the director is clearly asymmetric about a point $2 \mu \mathrm{m}$ into the cell with the homogeneous section of the cell underdriven and the homeotropic overdriven. On removal of the voltage the director steadily relaxes to the $0 \mathrm{~V}$ equilibrium state over a further $6 \mathrm{~ms}$ [Fig. 3(c)] and again, the effect of the rate of change of the applied field is demonstrated by the immediate optical response of the cell.

It should be noted that for this study of the potential for the driving both on and off of a hybrid aligned cell containing a dual-frequency liquid crystal, no optimization of either the material, the shape of the voltage pulse or the cell structure has been undertaken, and yet the entire on-off switching time $(\approx 19 \mathrm{~ms})$ is still much faster than the comparable switching time for a standard nematic HAN cell being switched on and allowed to relax back to its $0 \mathrm{~V}$ equilibrium state $(\approx 65 \mathrm{~ms}) .{ }^{14}$ However, before the dual-frequency HAN can be considered as a contender for use as a display device the problem of temperature dependence of the dielectric anisotropy must be overcome.

In conclusion, the rapidly changing dynamic director profile of a dual-frequency HAN cell driven from $0 \mathrm{~V}$ to homeotropic alignment and back again by using a multifrequency sinusoidal pulse has been measured. The new timeresolved FLGM technique, collecting data on a $2 \mu$ s time scale, has allowed the rapid response of the cell to changes in applied frequency to be observed optically and allowed the complex form of the director profile during the entire reorientation process to be measured in exquisite detail.

This project forms part of a COMIT Faraday partnership and the authors wish to thank the Engineering and Physical Sciences Research Council and the DTI for the financial support of this work.

${ }^{1}$ G. P. Bryan-Brown, C. V. Brown, I. C. Sage, and V. C. Hui, Nature (London) 392, 365 (1998).

${ }^{2}$ S. A. Jewell and J. R. Sambles, J. Appl. Phys. 92, 1 (2002).

${ }^{3}$ S. A. Jewell and J. R. Sambles, Mol. Cryst. Liq. Cryst. 401, 181 (2003).

${ }^{4}$ E. P. Raynes and I. Shanks, Electron. Lett. 10, 114 (1974).

${ }^{5}$ M. Schadt, Annu. Rev. Mater. Sci. 27, 305 (1997).

${ }^{6}$ Y. Q. Lu, X. Liang, Y. H. Wu, F. Du, and S. T. Wu, Appl. Phys. Lett. 85, 16 (2004).

${ }^{7}$ S. A. Jewell, J. R. Sambles, J. W. Goodby, A. W. Hall, and S. J. Cowling, J. Appl. Phys. 95, 5 (2004).

${ }^{8}$ F. Yang, L. Ruan, and J. R. Sambles, J. Appl. Phys. 88, 11 (2000).

${ }^{9}$ L. Z. Ruan and J. R. Sambles, Phys. Rev. Lett. 90, 168701 (2003).

${ }^{10}$ F. Z. Yang and J. R. Sambles, J. Opt. Soc. Am. B 16, 3 (1999).

${ }^{11}$ N. J. Smith and J. R. Sambles, J. Appl. Phys. 85, 8 (1999).

${ }^{12}$ D. Y. K. Ko and J. R. Sambles, J. Opt. Soc. Am. A 5, 1863 (1988).

${ }^{13}$ S. A. Jewell and J. R. Sambles, Appl. Phys. Lett. 84, 1 (2004).

${ }^{14}$ S. A. Jewell and J. R. Sambles, Appl. Phys. Lett. 82, 19 (2003). 